\title{
Optical properties of as-grown and annealed InAs(N)/InGaAsP strained multiple quantum wells
}

\author{
Guan-Ru Chen, Hao-Hsiung Lin, ${ }^{a}$ Jyh-Shyang Wang, and Ding-Kang Shih \\ Department of Electrical Engineering, National Taiwan University, Room 419 Taipei, Taiwan, \\ Republic of China
}

(Received 2 January 2001; accepted for publication 24 September 2001)

\begin{abstract}
Optical and structural properties of as-grown and annealed $\operatorname{InAs}(\mathrm{N}) / \mathrm{InGaAsP}$ strained multiple quantum-well (MQW) structures grown by gas source molecular-beam epitaxy are investigated by photoluminescence (PL), double crystal x-ray diffraction, and photoconductivity spectroscopies. Properties of the as-grown and annealed MQW's are studied and those of the InAs/InGaAsP MQW (C821) and the InAsN/InGaAsP MQW with the lowest nitrogen contents $N=1.1 \%$ in the well (C822) are compared. For the C821 InAs/InGaAsP MQW with a very large total strain, a low energy shoulder, possibly induced by defects or impurities, can be seen in the low temperature PL spectrum, and a large density of nonradiative recombination centers is found. For nitrogen-containing MQW's, the PL full width at half maximum and PL peak evolutions with increasing annealing temperature are influenced by the alloy inhomogeneities. The initial redshift of the PL peak after rapid thermal annealing means that the luminescence is dominated by As-rich regions in these as-grown samples. Exciton localization induced by alloy disorders is also found in high-nitrogen-content samples. By comparing the experimental results of $\mathrm{C} 821$ and $\mathrm{C} 822$, adding a little nitrogen to reduce the rather large total strain in the structure is beneficial to structural and optical quality improvement. (C) 2001 American Institute of Physics. [DOI: 10.1063/1.1418422]
\end{abstract}

\section{INTRODUCTION}

Nitrogen-containing III-(V,N) semiconductors have long been considered as potential materials for optoelectronic applications. Several mixed group-V nitride alloys, such as $\mathrm{GaP}_{0.98} \mathrm{~N}_{0.02}$ and $\mathrm{GaAs}_{0.8} \mathrm{~N}_{0.2}$, were predicted by Vegard's law to be lattice matched to silicon, ${ }^{1}$ providing the possibility of integrating III-V semiconductors to silicon technology. However, most studies to date have focused on the group-III sublattice like InGaN and $\mathrm{AlGaN}$; there has been much less research on nitrides with mixing on the group-V sublattice. In recent years, III- $(\mathrm{V}, \mathrm{N})$ materials such as InGaAsN and GaAsN have been successfully grown by molecular-beam epitaxy $(\mathrm{MBE})^{1,2}$ and metalorganic vapor phase epitaxy $y^{3,4}$ lying inside the miscibility gap, thus motivated the study on these materials.

These mixed group- $\mathrm{V}$ nitrides have serious bowing effects because of the large and local perturbation of the crystal lattice potential resulting from the replacement of atoms such as As or $\mathrm{P}$ with much smaller and more electronegative $\mathrm{N}$ atoms. ${ }^{5-7}$ For the midinfrared $2-5 \mu \mathrm{m}$ wavelength applications, InAsN alloy could be a very promising material, but so far there have been only few studies on it. ${ }^{8-11}$ We had recently demonstrated a high-quality $2.2 \mu \mathrm{m}$ InAs/InGaAs/InP strained multiple quantum-well (MQW) laser grown by gas source MBE (GSMBE). ${ }^{12}$ Replacing InAs with InAsN can ease the critical thickness limitation on the quantum-well (QW) because of the smaller compressive strain and can also further reduce the QW band gap energy owing to the large bowing effect of InAsN, thus pushing the wavelength of la-

${ }^{\text {a)} E l e c t r o n i c ~ m a i l: ~ h h l i n @ c c . e e . n t u . e d u . t w ~}$ sers on InP substrates to the longer infrared range. In this article, the optical and structural properties of as-grown and annealed $\operatorname{InAs}(\mathrm{N}) / \mathrm{InGaAsP}$ strained MQW structures grown by GSMBE will be investigated by photoluminescence (PL), double crystal x-ray diffraction (DXRD), and photoconductivity (PC) spectroscopies. The effects of rapid thermal annealing (RTA) will be discussed and the properties of samples without and with nitrogen contents will be compared.

\section{EXPERIMENTS}

All MQW structures studied in this article were grown on (001) InP substrates by a VG V-80 H GSMBE system. Active nitrogen species were provided by an EPI UNI-bulb rf plasma source. First, a $0.5 \mu \mathrm{m}$ thick $\operatorname{InGaAsP}(\lambda=1.3 \mu \mathrm{m})$ lattice matched to InP was grown at a $2 \mu \mathrm{m} / \mathrm{hr}$ growth rate to serve as the buffer layer. Then, the rf plasma power was fixed at $120 \mathrm{~W}$ and the substrate temperature was set to $400^{\circ} \mathrm{C}$ to grow the MQW structure. The MQW's consisted of ten periods of QW structures with $30 \AA \operatorname{InAs}(\mathrm{N})$ well and $200 \AA$ $\operatorname{InGaAsP}(\lambda=1.3 \mu \mathrm{m}$, lattice matched to an $\mathrm{InP})$ barrier; the $\operatorname{InAs}(\mathrm{N})$ layer growth rate was $1.5 \mu \mathrm{m} / \mathrm{h}$, and the As/In ratio was two. No growth interruption was used at the heterointerfaces. To improve the sample quality, postgrowth RTA treatments were used and several different conditions were tried. The annealing temperatures ranged from $500^{\circ} \mathrm{C}$ to $575^{\circ} \mathrm{C}$ and the annealing duration was fixed at $15 \mathrm{~min}$. Samples were put up-side down in contact with the Si wafer to prevent desorption of the group- $\mathrm{V}$ elements. All annealing processes were carried out in a nitrogen atmosphere. 
PL, PC, and DXRD spectroscopies were used for the investigation of the optical and structural properties of our MQW samples. Samples for PL measurements were mounted on the cold finger of a variable temperature and closed-cycle helium cryostat and excited by the $514.5 \mathrm{~nm}$ line of an argon ion laser. The luminescence was collected by two $\mathrm{CaF}_{2}$ condenser lens and dispersed by a SPEX 500M monochromator into an liquid-nitrogen-cooled HAMAMATSU P3357-02 InSb photodiode. A general lock-in technique was used in our PL measurements. Both temperature-dependent and excitation-power-dependent PL measurements were carried out to investigate the optical properties of our as-grown and annealed samples. As for the PC spectrum, samples used for this measurement were MQW's with two indium contacts on the surface and were bonded on ceramic pads. The pad was mounted on the cold finger inside a cryostat. The infrared (IR) light source of different wavelengths was provided by a Perkin-Elmer Spectrum 2000 Fourier transform IR (FTIR) spectrometer and normally incident into the MQW. Photogenerated carriers were collected through the indium contacts by a Stanford Research SR570 low-noise current preamplifier by which the bias was applied, too. The amplified photocurrent was transformed into voltage signal and sent into the FTIR spectrometer. Both SR570 and the FTIR system were controlled by a personal computer. The photoconductivity spectrum was thus obtained and could be viewed as an equivalent absorption spectrum. Since the bias was applied laterally, no Stark effect happened. The DXRD spectroscopy was measured by a Bede Scientific QC1a diffractometer. The nitrogen compositions of the well layers were determined from the DXRD spectra fittings using commercial dynamic simulation software. MQW C821 contains no nitrogen contents and the nitrogen compositions of MQW's C822, C823, and C824 are 1.1\%, 3.5\%, and $5.9 \%$, respectively.

\section{RESULTS AND DISCUSSION}

\section{A. Properties of the as-grown and annealed MQW's}

The full width at half maximum (FWHM) of x-ray Bragg diffraction peaks is usually used as an indicator of interface uniformity and the degree of crystallinity. InAs/ InGaAsP MQW C821 has very large total strain owing to the $3.2 \%$ mismatch between InAs and InP, but according to the (400) DXRD spectra of C821 as-grown and annealed samples, no obvious strain relaxation occurs and the spectra are almost the same for all annealing temperatures, as shown in Fig. 1. However, it can still be identified that the sharpest peaks are achieved at an annealing temperature $T_{a}$ $=525^{\circ} \mathrm{C}$, which is the optimum annealing condition according to the PL measurement results illustrated in Fig. 2. By comparing the (400) and (511) XRD spectra of C821, an in-plane lattice mismatch $(\Delta a / a)_{\|}=5.93 \times 10^{-4}$ is obtained, indicating a slight degree of relaxation. Figure 2(a) shows the PL data of this series of samples measured at $T=12 \mathrm{~K}$. The PL profile of C821 (as grown) has a shoulder at the low energy side and a long tail extending into lower energy. This could be attributed to defect- or impurity-related luminescence. ${ }^{13-15}$ After $500{ }^{\circ} \mathrm{C}$ annealing, the intensity of

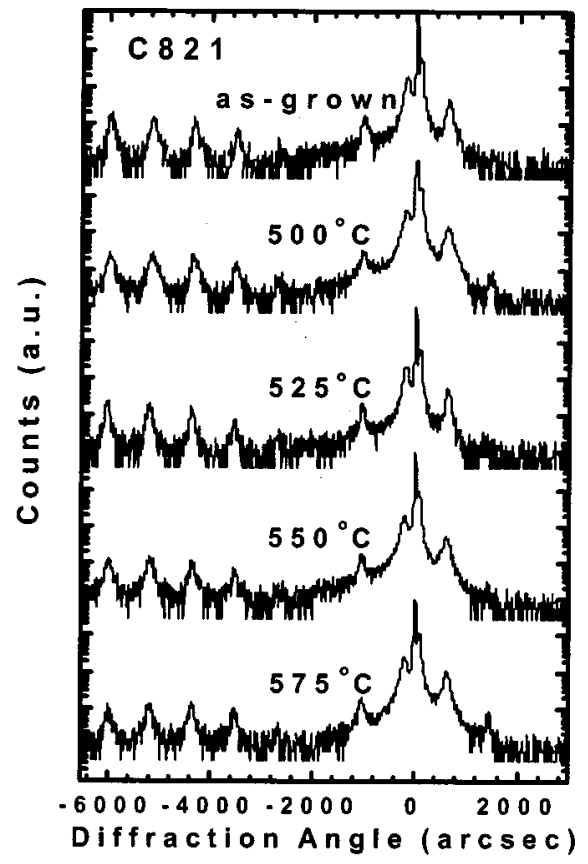

FIG. 1. (400) XRD spectra of C821 annealed at different temperatures.

impurity- or defect-related luminescence somehow increases more than that of the main peak. The position of the main peak is still fixed under this annealing condition, indicating that the interdiffusion between well and barrier is not activated yet. ${ }^{16,17}$ A relatively larger increase of the intensity of the low energy shoulder might be due to the elimination of higher-density nonradiative recombination centers around these impurities or defects. For $T_{a}=525^{\circ} \mathrm{C}$, the PL intensity is obviously enhanced and the defect-related luminescence is suppressed. The PL peak position is blueshifted a little, which means that the interdiffusion process is activated. The shift of PL peak energy increases with increasing $T_{a}$, along with a decreasing defect- or impurity-related luminescence. A fairly symmetric line shape of the $575^{\circ} \mathrm{C}$ annealed sample indicates that the defects or impurities responsible for the low energy shoulder are completely annealed out. Integrated PL intensities at different $T_{a}$ 's are shown in Fig. 2(b). The intensity first rises and then falls with increasing $T_{a}$. The dropping of PL intensity might be caused by the thermally generated defects acting as nonradiative recombination centers. The optimum annealing temperature is $525^{\circ} \mathrm{C}$ and the intensity of the optimum-condition-annealed sample is about six times the value of the as-grown one. This large degree of improvement [much larger than observed in the InAs/ InGaAs single $\mathrm{QW}]^{11}$ implies that there exists a lot of nonradiative recombination centers in C821 (as grown), possibly due to the large total strain.

Now, we turn the discussion into nitrogen-containing samples. X-ray rocking curves of C822 under different annealing conditions are shown in Fig. 3. After annealing, the originally unclear peak situated around $-5000^{\prime \prime}$ becomes more obvious because of improved crystallinity. The peak positions remain unchanged after annealing, showing no loss of the $\mathrm{N}$ or As atoms. It should be noticed, however, that the Bragg peaks of the $575^{\circ} \mathrm{C}$ annealed sample are broadened. 

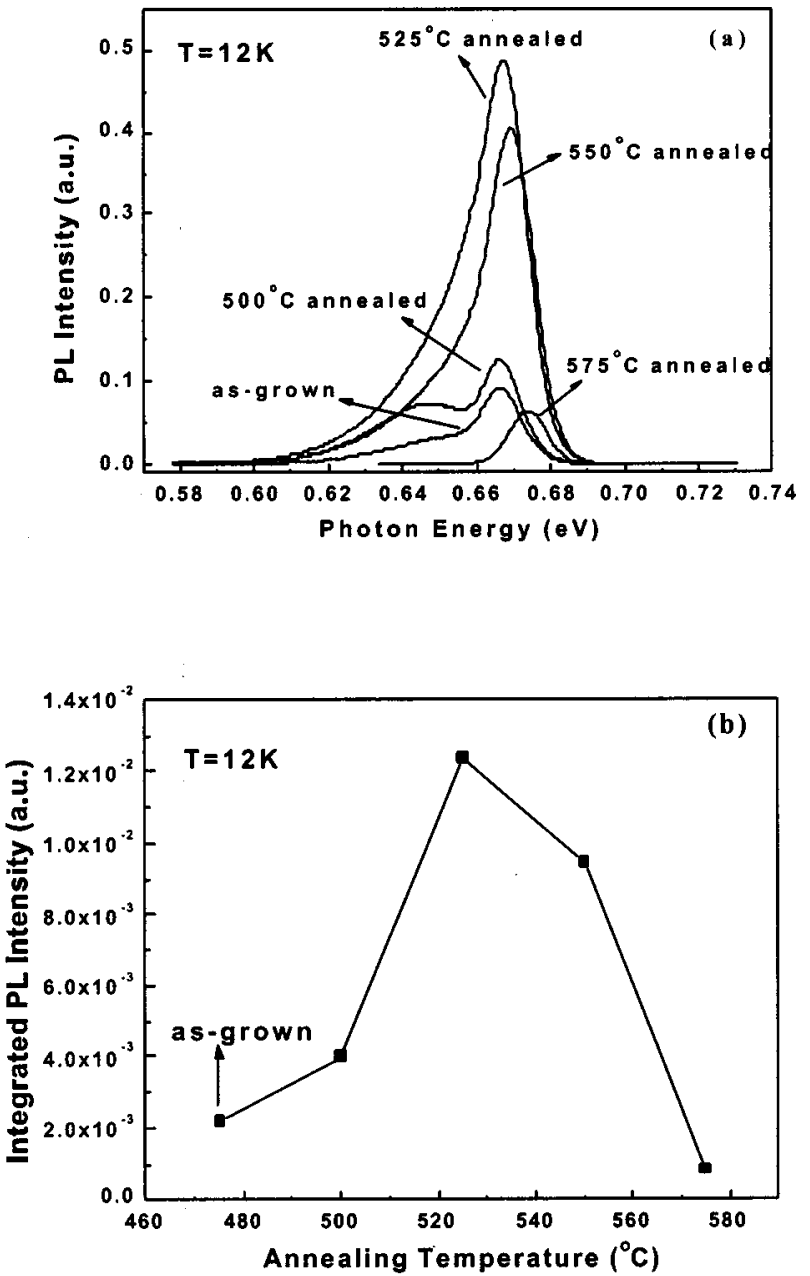

FIG. 2. (a) PL spectra of C821 as-grown and annealed samples measured at $T=12 \mathrm{~K}$. (b) Integrated PL intensity at $T=12 \mathrm{~K}$ of C 821 as-grown and annealed samples.

This may be due to a degraded crystallinity induced by local strain relaxation. PL spectra measured at $T=11 \mathrm{~K}$ and the corresponding integrated intensities and FWHM's of all the samples are illustrated in Fig. 4. No apparent phase separation appears, but a low energy tail still exists. The existence of band-tail states and the broad line shape represent that alloy disorders play an important role in InAsN material. The optimum annealing temperature of $\mathrm{C} 822$ is $525^{\circ} \mathrm{C}$, as can be seen in Fig. 4(b), and the PL FWHM of C822 is obviously reduced after $500^{\circ} \mathrm{C}$ annealing and reaches a minimum value at $T_{a}=525^{\circ} \mathrm{C}$. For $T_{a}>525^{\circ} \mathrm{C}$, its value almost does not change with rising $T_{a}$, indicating that the material uniformity can not be improved further by higher $T_{a}$ 's. An anomalous PL peak shift after annealing is observed for nitrogencontaining MQW's. This strange behavior can not be clearly identified in $\mathrm{C} 822$ with only $1.1 \%$ nitrogen composition but is obviously observed in $\mathrm{C} 823(N=3.5 \%)$, as shown in Fig. 5(a). The PL is probably dominated by As-rich regions before annealing since the PL peak is first redshifted after RTA treatment. Possible mechanisms responsible for this are the As diffusion out of As-rich regions and the reduced nonradiative recombination centers inside $\mathrm{N}$-rich regions. The following blueshift could be ascribed to the interdiffusion pro-

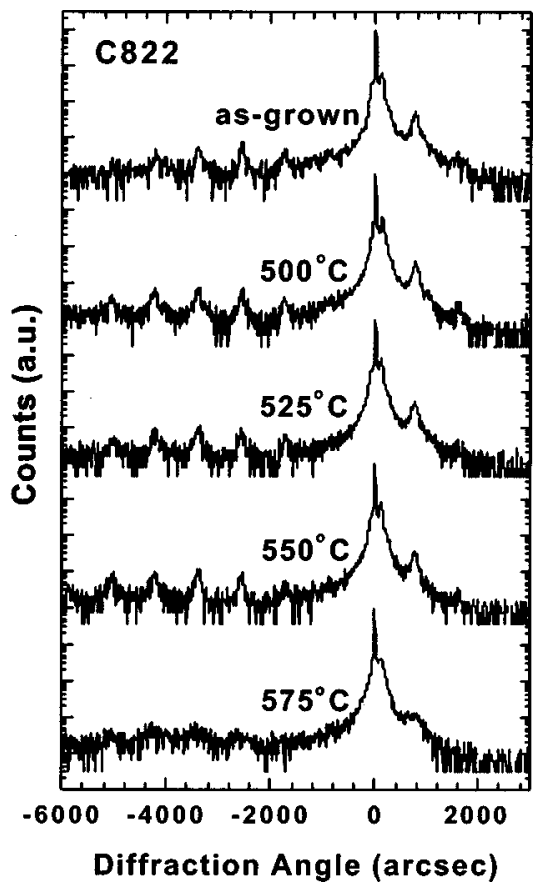

FIG. 3. (400) XRD spectra of C822 annealed at different temperatures.
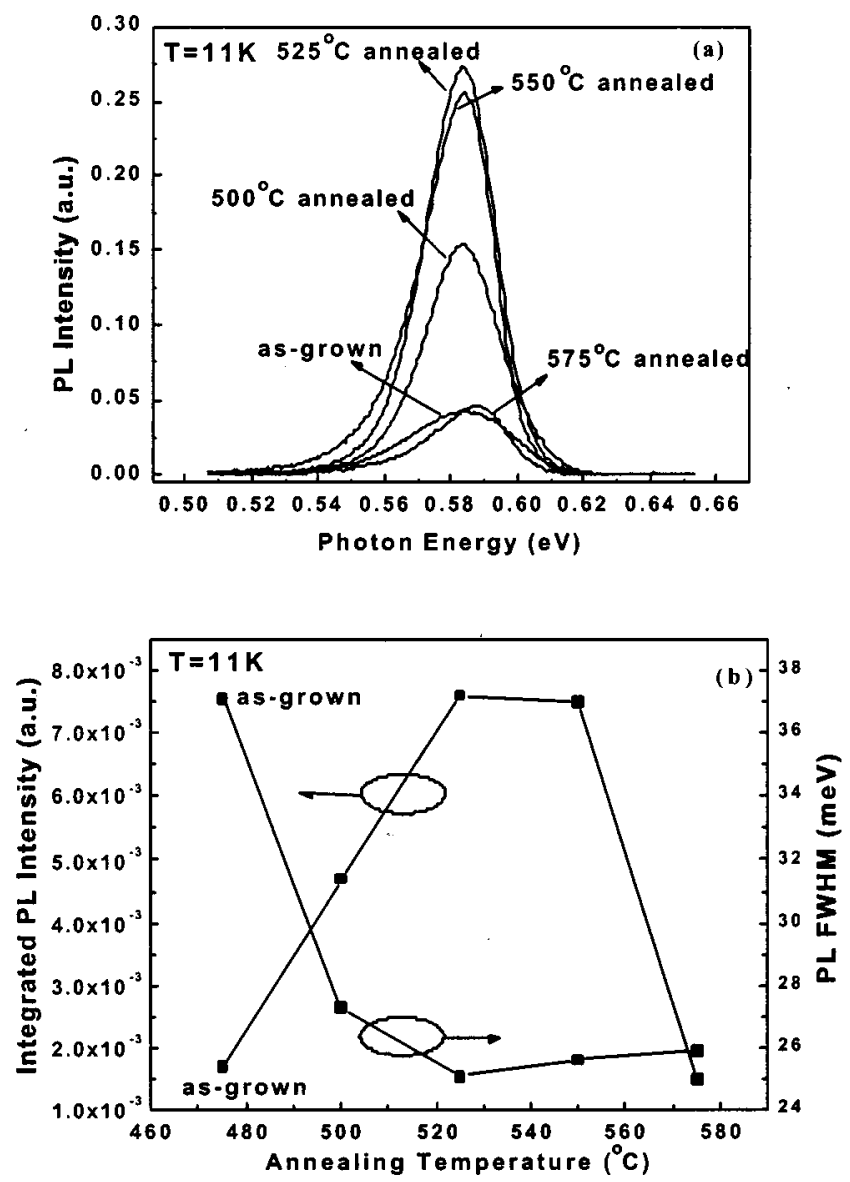

FIG. 4. (a) PL spectra of C822 as-grown and annealed samples measured at $T=11 \mathrm{~K}$. (b) $11 \mathrm{~K}$ integrated PL intensity and PL FWHM of C822 as-grown and annealed samples. 

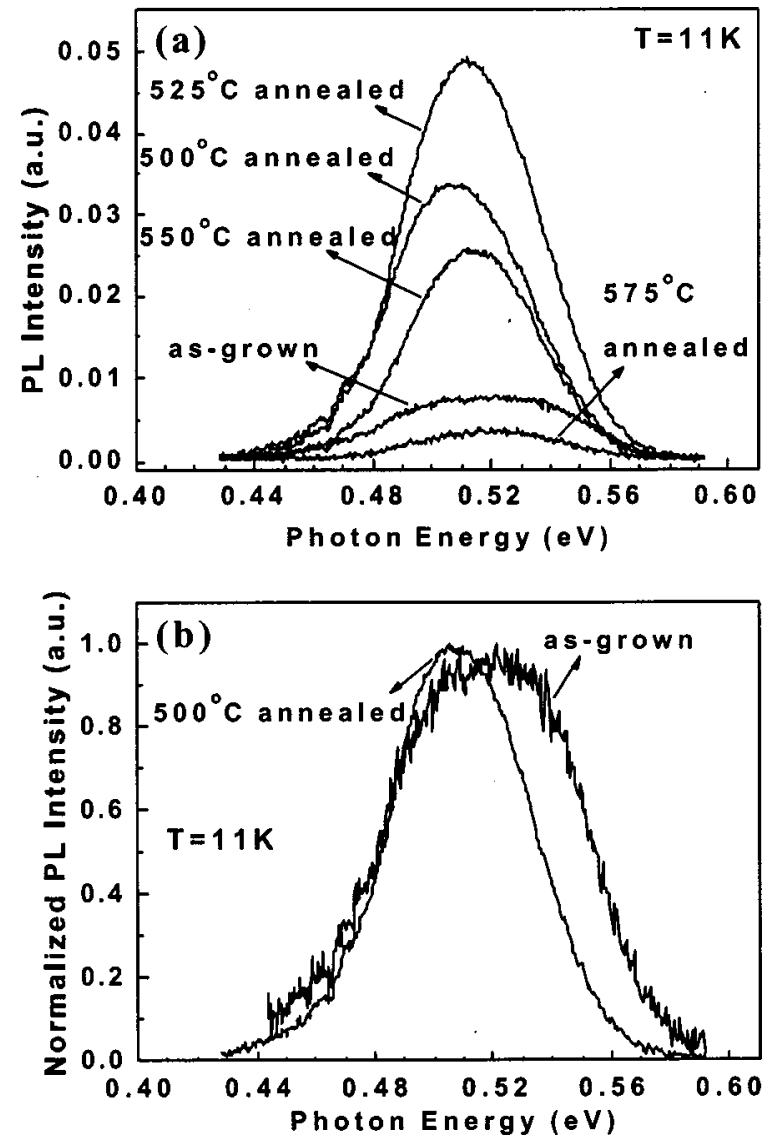

FIG. 5. (a) PL spectra of C823 as-grown and annealed samples measured at $T=11 \mathrm{~K}$. (b) Normalized PL spectra of C823 as-grown and $500{ }^{\circ} \mathrm{C}$ annealed samples.

cess between well and barrier, which is activated at higher annealing temperatures. From the normalized spectra of C823 (as-grown) and C823 $\left(500^{\circ} \mathrm{C}\right)$ shown in Fig. 5(b), the argument that the luminescence of the nitrogen-containing as-grown samples originate mainly from As-rich regions is reasonable and able to properly explain the observed redshift. DXRD spectra, PL FWHM's and integrated PL intensities of C823 at different $T_{a}$ 's all exhibit trends similar with those of C822. For C824 with $N=5.9 \%$ in the well layer, the characteristic of DXRD spectra shown in Fig. 6 is a little different from that of $\mathrm{C} 822$ and $\mathrm{C} 823$. There is no obvious degradation of the $575^{\circ} \mathrm{C}$ spectrum, indicating that the structure is more stable than C822 and C823. This higher thermal stability might be due to the higher nitrogen contents, which increase the average bond strength. According to the PL measurement results (data not shown), the PL FWHM of C824 after annealing is still larger than $60 \mathrm{meV}$, therefore, a very strong alloy inhomogeneity effect is expected to exist and is very difficult to eliminate. It can also be found that the enhancement of PL intensity after RTA is limited due to a very large density of nonradiative centers. The optimum annealing temperature of $\mathrm{C} 824$ is $500^{\circ} \mathrm{C}$, which is lower than that of $\mathrm{C} 822$ and $\mathrm{C} 823$. It seems that the optimum $T_{a}$ value decreases with increasing nitrogen composition by comparing the PL intensity evolution with $T_{a}$ of C822-C824. PC spectra of optimum-condition-annealed C823 and C824 along with low temperature PL spectra are shown in Fig. 7.

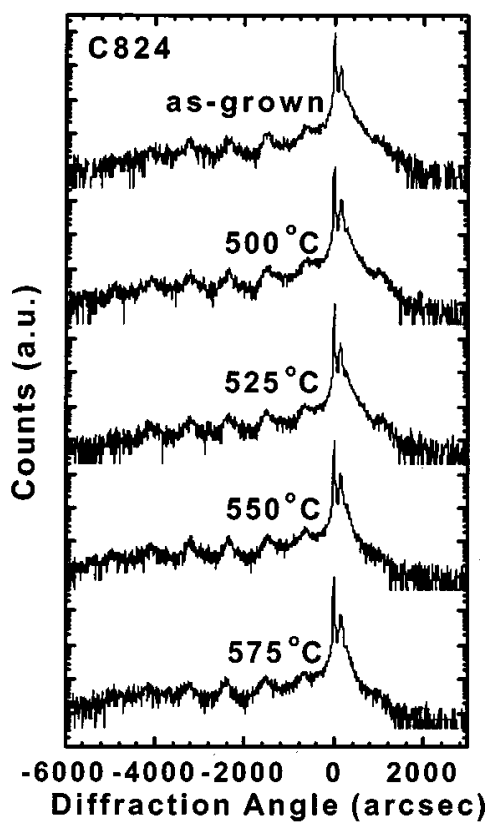

FIG. 6. (400) XRD spectra of C824 annealed at different temperatures.

For these two samples with high-nitrogen contents in well layers, the exciton localization effect is very obvious since manifest Stokes shifts can be observed. ${ }^{13,18}$ The absorption edge of $\mathrm{C} 824$ is flatter due to the less definite absorption
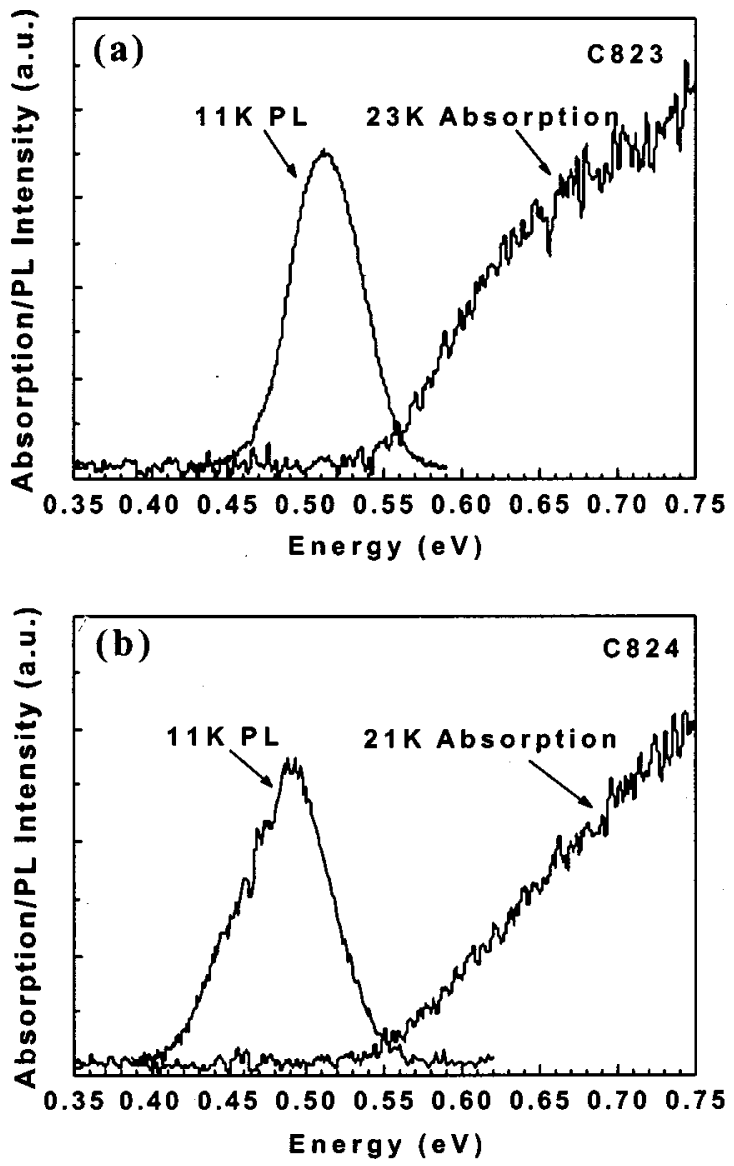

FIG. 7. (a) $11 \mathrm{~K}$ PL and $23 \mathrm{~K}$ PC spectra of $\mathrm{C} 823\left(525^{\circ} \mathrm{C}\right.$ annealed). (b) 11 $\mathrm{K}$ PL and $21 \mathrm{~K}$ PC spectra of $\mathrm{C} 824\left(500^{\circ} \mathrm{C}\right.$ annealed). 

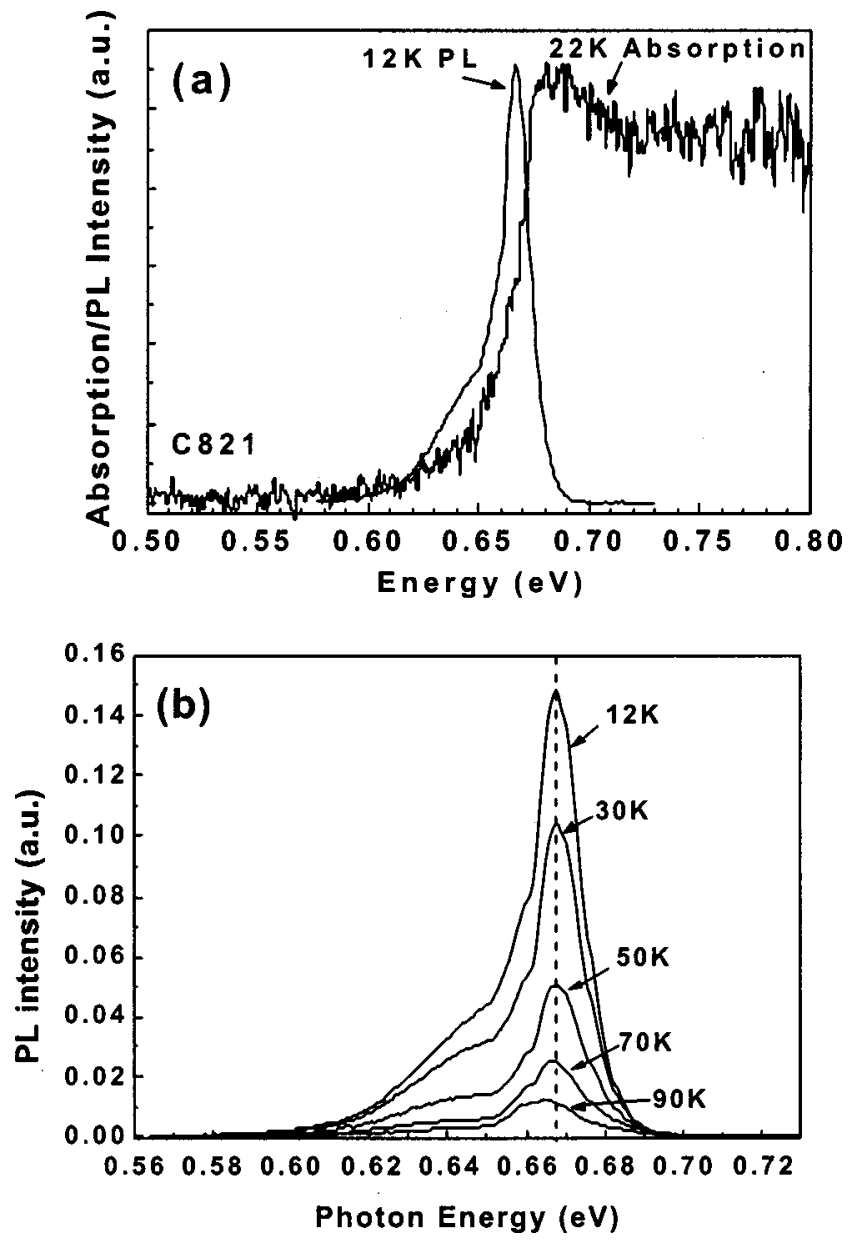

FIG. 8. (a) Temperature-dependent PL spectra of C821 (as-grown) from 12 $\mathrm{K}$ to $90 \mathrm{~K}$. (b) Comparison of $12 \mathrm{~K} \mathrm{PL}$ and $22 \mathrm{~K} \mathrm{PC}$ spectra of C821 (as grown).

edge caused by alloy inhomogeneity, but the position seems approximately unchanged compared with the edge of C823. The PL peak of C824 is redshifted (but not much) with respect to that of $\mathrm{C} 823$, and the whole PL profile extends further into low energy. The rather large PL FWHM and long extension of absorption edge imply that these high-nitrogencontents samples possess characteristics somewhat like amorphous semiconductor materials.

\section{B. Comparison of $\mathrm{C} 821$ and $\mathrm{C} 822$}

The temperature-dependent PL spectra of C821 (as grown) are shown in Fig. 8(a). It can be seen that the PL peak energy is fixed up to $T=50 \mathrm{~K}$, and the defect- or impurity-related luminescence gradually disappears with rising temperature. For comparison, the $22 \mathrm{~K}$ absorption spectrum and the $12 \mathrm{~K} \mathrm{PL}$ spectrum are plotted in Fig. 8(b). It is obvious that the PL comes from localized states. Here the 12 K PL spectrum is supposed to be almost identical to the $22 \mathrm{~K}$ one. A Stokes shift is clearly identified and might come from the localization of excitons induced by partial relaxation of this highly strained structure. ${ }^{13}$ The band-filling behavior observed in the excitation-power-dependent PL spectra also verifies this argument. With the addition of a little nitrogen, the localization effect induced by large total strain seems to
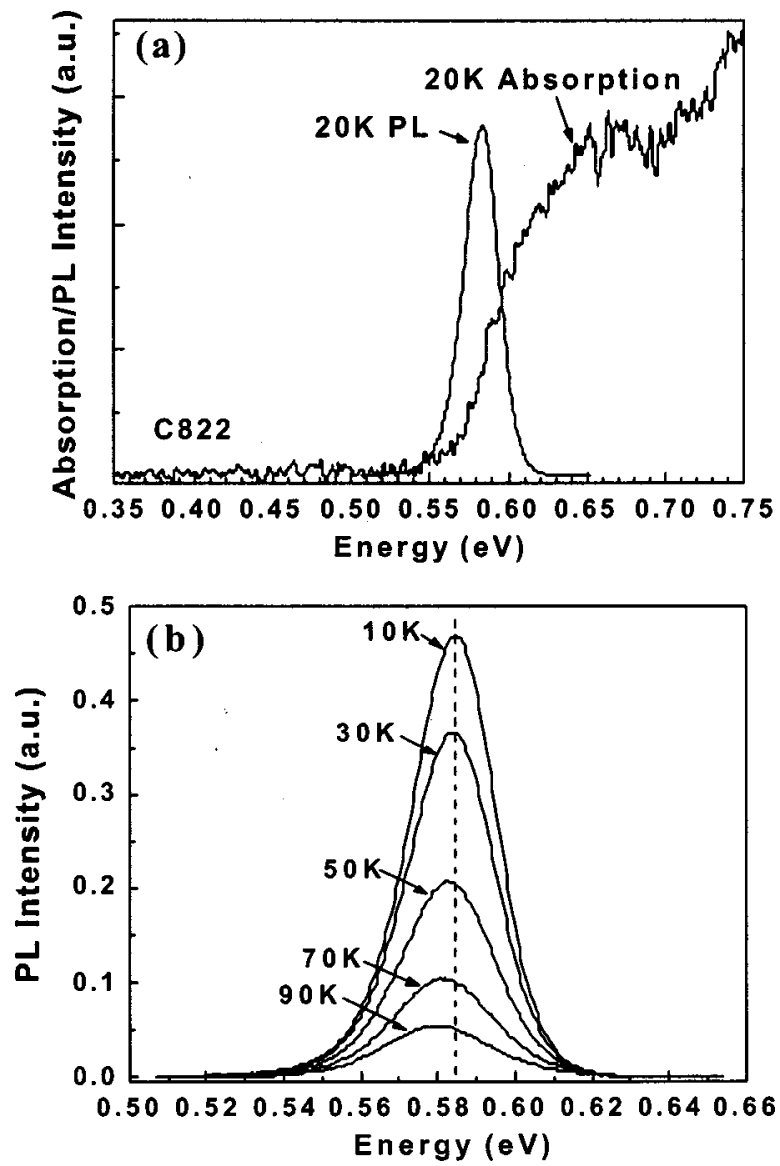

FIG. 9. (a) $20 \mathrm{~K} \mathrm{PL}$ and PC spectra of $\mathrm{C} 822\left(525^{\circ} \mathrm{C}\right.$ annealed). (b) Temperature-dependent PL spectra of C822 $\left(525^{\circ} \mathrm{C}\right.$ annealed) from 10 to 90 $\mathrm{K}$.

be reduced. Localization of excitons is not obvious from the comparison of $20 \mathrm{~K} \mathrm{PL}$ and absorption spectra of C822 $\left(525^{\circ} \mathrm{C}\right.$ annealed) shown in Fig. 9(a), and the PL peak begins to redshift at a very low temperature as illustrated in Fig. 9(b), which is a consequence of reduced exciton localization effect. The previously mentioned localization effect induced by partial relaxation is now eliminated by the introduction of nitrogen, and the exciton localization resulting from alloy disorders is not obvious under this low-nitrogen-contents condition. The sharpness of the absorption edge shown in Fig. 9(b) clearly decreases compared with C821 (N-free sample). We ascribe this to the less definite absorption edge due to alloy inhomogeneity.

$\alpha$ values extracted from the relation $I_{\mathrm{PL}} \propto I_{\mathrm{ex}}^{\alpha}$, where $I_{\mathrm{PL}}$ is the integrated PL intensity and $I_{\mathrm{ex}}$ is the excitation power intensity, are useful for one to evaluate the sample quality. According to Fouquet et al., ${ }^{19} \alpha=1$ if the radiative recombination process is excitonic, and $\alpha=2$ represents a bimolecular free carrier recombination process and meanwhile nonradiative recombination dominates. $\alpha$ values of C821 and C822 after optimum-condition annealing at four different temperatures are summarized in Table I. In the highly strained MQW C821, even after RTA treatment, the recombination process starts to be dominated by free carriers rather than excitons for $T>140 \mathrm{~K}$. Therefore, a larger number of nonradiative recombination centers which are easily acti- 
TABLE I. $\alpha$ values of $525^{\circ} \mathrm{C}$-annealed C821 InAs/InGaAsP MQW and C822 InAsN/InGaAsP MQW extracted from the relation $I_{\mathrm{PL}} \propto I_{\mathrm{ex}}^{\alpha}$.

\begin{tabular}{ccc}
\hline \hline Temperature $(\mathrm{K})$ & $\alpha$ 's of C821 & $\alpha$ 's of C822 \\
\hline 11 & $0.85 \pm 0.01$ & $0.90 \pm 0.03$ \\
80 & $1.19 \pm 0.02$ & $1.05 \pm 0.04$ \\
140 & $1.46 \pm 0.03$ & $1.23 \pm 0.03$ \\
200 & $1.76 \pm 0.07$ & $1.33 \pm 0.04$ \\
\hline \hline
\end{tabular}

vated at a higher temperature are supposed to exist in C821. On the other hand, the increase of the $\alpha$ values of $\mathrm{C} 822$ with increasing temperature is not as intense as in C821, indicating a better quality by reducing the large total compressive strain.

\section{CONCLUSION}

PL, DXRD, and PC spectroscopies were used to investigate the properties of InAs(N)/InGaAsP MQW's. For the C821 InAs/InGaAsP MQW with very large total strain, a low energy shoulder possibly induced by defects or impurities can be seen in the low temperature PL spectrum, and the large degree of PL improvement after RTA implies the existence of a large number of defects acting as nonradiative recombination centers. For nitrogen-containing samples C822-C824, the PL FWHM and PL peak evolutions with increasing annealing temperature are influenced by the alloy inhomogeneities. The initial PL peak redshift of these MQW's after RTA means that the luminescence is dominated by As-rich regions in these as-grown samples. C824 MQW with highest nitrogen composition $(N=5.9 \%)$ seems to be thermally more stable since XRD spectra of samples annealed at different temperatures are approximately the same. C823 and C824 with higher nitrogen contents are found to possess characteristics somewhat like amorphous semiconductor materials. By comparing the experimental results of C821 and C822, with the introduction of a little nitrogen into the well layer, the exciton localization is reduced, and the nonradiative recombination process becomes less serious, indicating that adding a little nitrogen to reduce the rather large total strain is beneficial to structural and optical quality improvement.

\section{ACKNOWLEDGMENT}

This work was supported by the National Science Council of the Republic of China under Contract No. NSC 892215-E-002-034.

${ }^{1}$ M. Kondow, K. Uomi, K. Hosomi, and T. Mozume, Jpn. J. Appl. Phys., Part 2 33, L1056 (1994).

${ }^{2}$ M. Kondow, T. Kitatani, S. Nakatsuka, M. C. Larson, K. Nakahara, Y. Yazawa, M. Okai, and K. Uomi, IEEE J. Sel. Top. Quantum Electron. 3, 719 (1997).

${ }^{3}$ S. Francoeur, G. Sivaraman, Y. Qiu, S. Nikishin, and H. Temkin, Appl. Phys. Lett. 72, 1857 (1998).

${ }^{4}$ S. Sato, Y. Osawa, and T. Saitoh, Jpn. J. Appl. Phys., Part 1 36, 2671 (1997).

${ }^{5}$ S. Sakai, Y. Ueta, and Y. Teauchi, Jpn. J. Appl. Phys., Part 1 32, 4413 (1993).

${ }^{6}$ S. H. Wei and A. Zunger, Phys. Rev. Lett. 76, 664 (1996).

${ }^{7}$ L. Bellaiche, S. H. Wei, and A. Zunger, Phys. Rev. B 54, 17568 (1996).

${ }^{8}$ H. Naoi, Y. Naoi, and S. Sakai, Solid-State Electron. 41, 319 (1997).

${ }^{9}$ R. Beresford, K. S. Stevens, and A. F. Schwartzman, J. Vac. Sci. Technol. B 16, 1293 (1998).

${ }^{10}$ M. Hao, S. Sakai, T. Sugahara, T. S. Cheng, and C. T. Foxon, J. Cryst. Growth 189, 481 (1998).

${ }^{11}$ J. S. Wang and H. H. Lin, J. Vac. Sci. Technol. B 17, 1997 (1999).

${ }^{12}$ J. S. Wang, H. H. Lin, and L. W. Sung, IEEE J. Quantum Electron. 34, 1959 (1998)

${ }^{13}$ A. Aït-Ouali, R. Y.-F. Yip, J. L. Brebner, and R. A. Masut, J. Appl. Phys. 83, 3153 (1998).

${ }^{14}$ E. Tournié, K. H. Ploog, and C. Alibert, Appl. Phys. Lett. 61, 2808 (1992).

${ }^{15}$ S. T. Davey, E. G. Scott, B. Wakefield, and G. J. Davies, Semicond. Sci. Technol. 3, 365 (1988).

${ }^{16}$ A. Saher Helmy, J. S. Aitchison, and J. H. Marsh, Appl. Phys. Lett. 71, 2998 (1997).

${ }^{17}$ Q. J. Xing, Solid State Commun. 98, 1009 (1996).

${ }^{18}$ I. A. Buyanova, W. M. Chen, G. Pozina, J. P. Bergman, B. Monemar, H. P. Xin, and C. W. Tu, Appl. Phys. Lett. 75, 501 (1999).

${ }^{19}$ J. E. Fouquet and A. E. Siegman, Appl. Phys. Lett. 46, 280 (1985). 